\title{
TALYS/TENDL verification and validation processes: Outcomes and recommendations
}

\author{
Michael Fleming ${ }^{1, \text { a }}$, Jean-Christophe Sublet ${ }^{1}$, Mark R. Gilbert ${ }^{1}$, Arjan Koning ${ }^{2}$, and Dimitri Rochman ${ }^{3}$ \\ 1 United Kingdom Atomic Energy Authority, Culham Science Centre, Abingdon, Oxfordshire, UK \\ 2 Nuclear Data Section, IAEA, Vienna, Austria \\ ${ }^{3}$ Reactor Physics and Systems Behavior Laboratory, Paul Scherrer Institute, Villigen, Switzerland
}

\begin{abstract}
The TALYS-generated Evaluated Nuclear Data Libraries (TENDL) provide truly general-purpose nuclear data files assembled from the outputs of the T6 nuclear model codes system for direct use in both basic physics and engineering applications. The most recent TENDL-2015 version is based on both default and adjusted parameters of the most recent TALYS, TAFIS, TANES, TARES, TEFAL, TASMAN codes wrapped into a Total Monte Carlo loop for uncertainty quantification. TENDL-2015 contains complete neutron-incident evaluations for all target nuclides with $\mathrm{Z} \leq 116$ with half-life longer than 1 second (2809 isotopes with 544 isomeric states), up to $200 \mathrm{MeV}$, with covariances and all reaction daughter products including isomers of halflife greater than 100 milliseconds. With the added High Fidelity Resonance (HFR) approach, all resonances are unique, following statistical rules. The validation of the TENDL-2014/2015 libraries against standard, evaluated, microscopic and integral cross sections has been performed against a newly compiled UKAEA database of thermal, resonance integral, Maxwellian averages, $14 \mathrm{MeV}$ and various accelerator-driven neutron source spectra. This has been assembled using the most up-to-date, internationally-recognised data sources including the Atlas of Resonances, CRC, evaluated EXFOR, activation databases, fusion, fission and MACS. Excellent agreement was found with a small set of errors within the reference databases and TENDL-2014 predictions.
\end{abstract}

\section{Introduction}

The UKAEA has a long history of supporting the development of new nuclear technologies and currently hosts the world's largest magnetic confinement nuclear reactor, JET. In order to meet the simulation demands for emerging and next-generation technologies, new technologies are required to extend our capabilities and anticipate challenges - including those of nuclear data and activation-inventory simulation. Over the past decade, the UKAEA has focused on the extension of its main nuclear observables code, FISPACT-II [1], developing it to best employ the rapidly maturing TALYS-generated Evaluated Nuclear Data Libraries (TENDL) for neutron and charged particle irradiation [2]. This has culminated in several verification and validation exercises for both FISPACT-II and its associated nuclear data libraries, covering a range of approaches summarised in this paper.

\section{Integro-differential fusion and accelerator data}

The production of an activation library which satisfies the various incident-neutron energy and materials requirements of the fission and fusion energy communities has involved many nuclear data experts. The previous European Activation Files (EAF) were the subject, in their continually evolving form, of several integral validation

$\overline{\text { a e-mail: michael.fleming@ukaea.uk }}$ efforts. Various contributors within the experimental and nuclear data communities provided comparisons between experimental and calculated heat or activity. By isolating a decay period where one nuclide dominates the measurement, the production of a specific radionuclide would be determined and the C/E ratio could be used to calculate the effective cross section for the reaction responsible for that product. A series of experiments have been performed and assembled for validation exercises with activation-level nuclear data libraries [3,4]. The remarkable development of the TENDL libraries, with eight distributions in as many years, has received the same effort that previous European Activation Files had enjoyed, leading to the UKAEA studies using FISPACT-II with TENDL and all other major nuclear data libraries on fusion decay heat [5].

This has been followed with a reanalysis of all the experiments considered in the legacy EAF validations, employing the complete TENDL reaction channels, upgraded FISPACT-II pathways algorithms, full variancecovariance treatment and various other improvements in a new UKAEA report [6]. These include a variety of neutron spectra including D-T, D-Li, D-Be, $p-\mathrm{D}_{2} \mathrm{O}$ and ${ }^{252} \mathrm{Cf}$. Time-dependent studies for the spectroscopic and total decay heats were performed, such as the ENEA Frascati Neutron Generator (FNG) nickel irradiation of Fig. 1. The work was completed alongside similar efforts for the FENDL-3.0/A libraries [7,8].

In a large number of cases, TENDL provides the only reliable nuclear data for all reaction channels and isomeric

(c) The Authors, published by EDP Sciences. This is an Open Access article distributed under the terms of the Creative Commons Attribution License 4.0 (http://creativecommons.org/licenses/by/4.0/). 


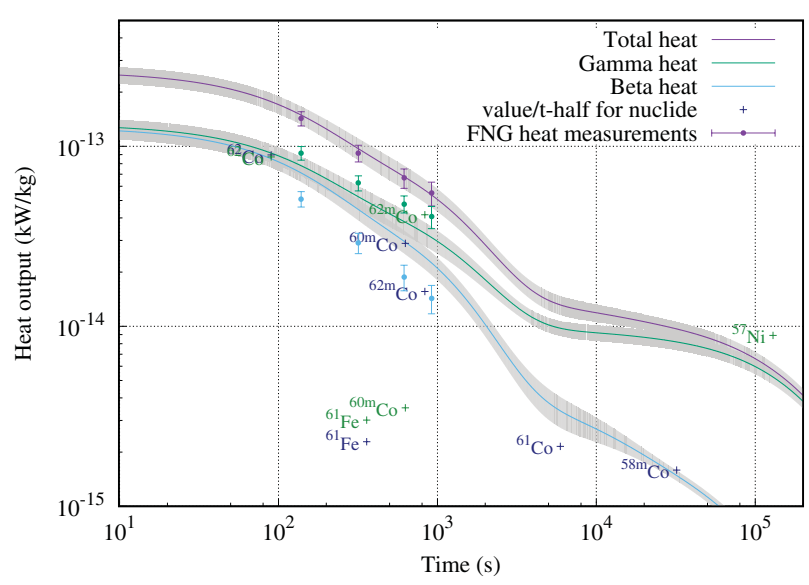

Figure 1. Total and spectroscopic decay heat measurements from an FNG irradiation of nickel with FISPACT-II predictions using TENDL-2014 [6]. Dominant nuclides are placed at coordinates that reflect their half-life and end-of-irradiation heat contributions.

branching ratios. These can be seen in cases such as nickel, where the absence of isomeric production cross sections result in significant under-prediction on the timeframe of minutes to hours, as demonstrated by the JAEA Fusion Neutron Source decay heat experiments, shown in Fig. 2. In all, approximately one third of all reaction channels considered in the validation were absent from the ENDF/B-VII.1, JENDL-4.0 and JEFF-3.2 libraries. Moreover, the distribution over all $\mathrm{C} / \mathrm{E}$ values from all integral comparisons with TENDL-2014 were superior to EAF-2007, with both a $20 \%$ increase in values between $0.8-1.2$ and a distribution average $\overline{C / E}$,

$$
\log (\overline{C / E})=\frac{1}{n} \sum_{i} \log \left(C_{i} / E_{i}\right),
$$

of 0.993 rather than the EAF-2007 value of 0.850. This indicates a systematic under-prediction in the EAF data which is intuitively due to the construction methodology: add reaction channels when and where required. The fundamental difference with TENDL, that all channels are considered equal in the eyes of the models and local parameters, provides a considerable improvement where nuclear data is expected beyond the regions of experimental support.

\section{Maxwellian-averaged cross sections}

Maxwellian-averaged cross sections (MACS) are essential to the understanding of stellar nucleosynthesis. Various nuclear interactions occur in stellar environments, resulting, for example, in the accumulation of high-Z elements and shaping the particular isotopic inventories that we observe in the universe. The importance of these cross sections has led to many measurements which probe MACS for multiple nuclides and energy regions. The reference used in a recent UKAEA report [9] was the KADoNiS database [10], largely taken from the work of Bao et al. [11]. The integral MACS were calculated using the well-known ENDF utility code inter, as well as the maxwav code developed at JAEA and used in a similar, earlier work [12]. In addition, the covariance data contained within TENDL-2014 was processed using

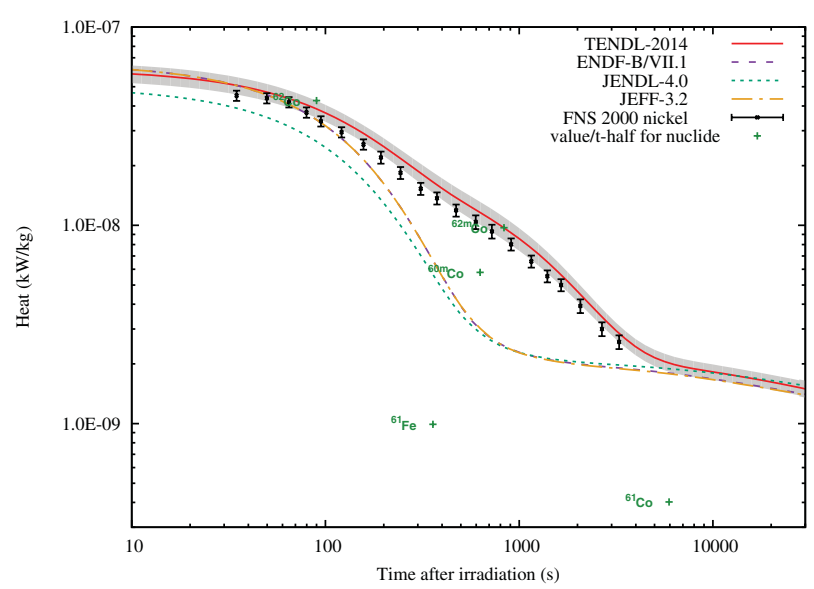

Figure 2. Total decay heat measurements from an JAEA FNS irradiation of nickel with FISPACT-II predictions using TENDL2014, ENDF/B-VII.1, JENDL-4.0 and JEFF-3.2 [5]. The lack of the metastable production for ${ }^{62} \mathrm{Co}$ results in a significant underprediction for well-known libraries.

FISPACT-II to calculate cross section uncertainties directly from the file.

One notable feature, the inclusion of statisticallyresolved high fidelity resonances (HFR) [13], allows an extension of the resolved resonances or a systematic generation of resonances where none would be available. The example of ${ }^{85} \mathrm{Kr}$, as shown in Fig. 3, demonstrates the potential of the added data to provide a more physical dataset.

Since TENDL provides all of the target nuclides for comparison with KADoNiS, the overall distribution of $\mathrm{C} / \mathrm{E}$ values is superior to those of the well-known (and similarly tested) ENDF/B-VII.1 and JENDL-4.0, particularly as approximately $20 \%$ of the targets are missing in the latter two.

\section{Residual production cross sections}

Above a few tens of $\mathrm{MeV}$, a great many reaction channels are open which increasingly 'overlap' just as (n,np) and $(\mathrm{n}, \mathrm{d})$. With a dozen or more nucleons ejected at some energies, the ENDF-6 channel-specific rules are clearly not applicable nor desirable. While the T6 calculations that generate the TENDL data follows all individual processes, these are summarised as total residual nuclide production, as reconstructed from the full $\mathrm{MF}=6$ emitted data into an equivalent $M F=10$. These are the exclusive channels above $30 \mathrm{MeV}$ and extend to $200 \mathrm{MeV}$ in the default TENDL, although higher-energy extensions exist. FISPACT-II has been designed specifically to process all of these data, shown for a few selected energies with ${ }^{120} \mathrm{Sn}$ as an example target in Fig. 4. The experimental data at higher energies for residual nuclide production typically include cumulative cross sections as the spray of radionuclides produced in spallation processes include many that decay over short time-scales and are not measured. To follow each of these cumulative nuclide yields, FISPACT-II has been employed to decay the precursors and calculate the cumulatives for each incident energy provided in the TENDL files. Taking natural tungsten as a target, an example for the production of hafnium isotopes with EXFOR data is shown in Fig. 5. The generation of all 

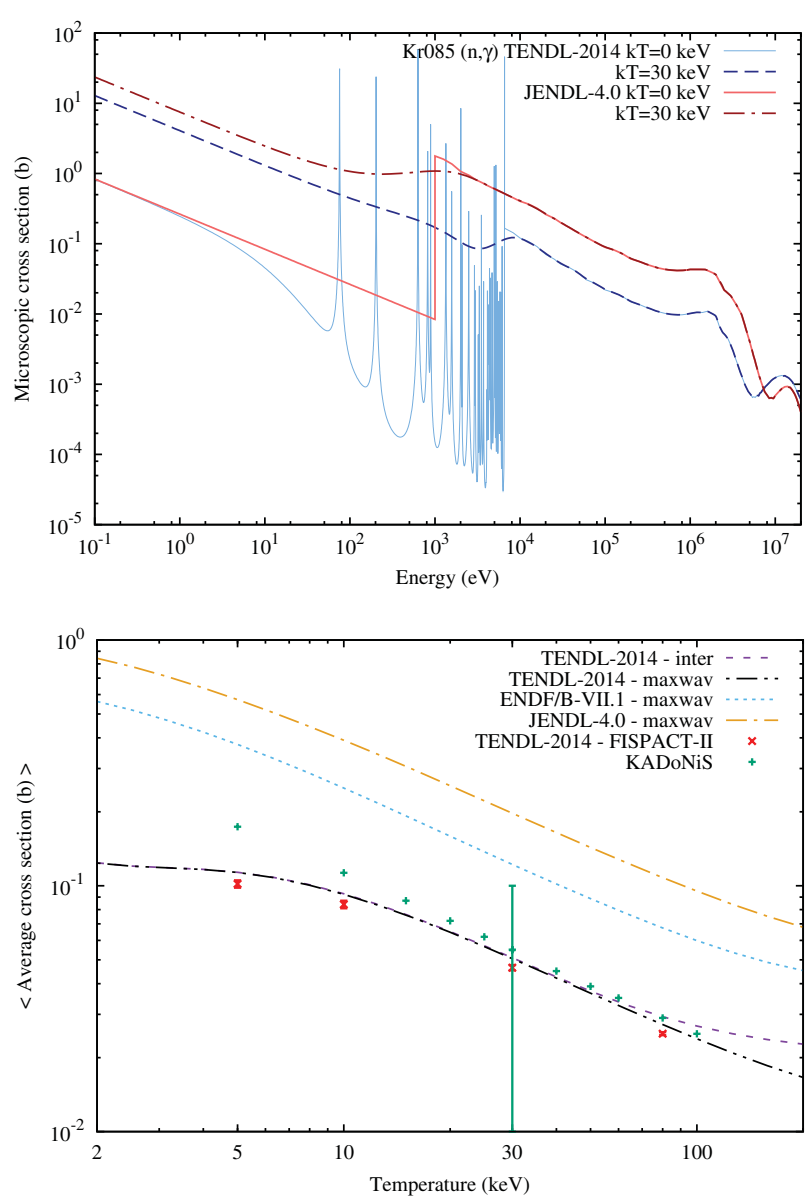

Figure 3. Neutron capture cross section for ${ }^{85} \mathrm{Kr}$ shown as microscopic with Doppler broadening for $\mathrm{kT}=30 \mathrm{keV}$ (top) and Maxwellian-averaged cross sections from multiple libraries (bottom).

products in each simulation at energies of several hundred $\mathrm{MeV}$ is computationally expensive and the incident energy grid is quite coarse, however the generation of complete tables of radionuclide production has several advantages as it is reproducible, easily tested and can be used without significant computational expense by activation codes such as FISPACT-II.

\section{Systematic trends in large libraries}

The complete nature of TENDL is a double-edged feature, offering robust capabilities far from the experimentally verified but also exposing less predictive regimes of the models or faults in input libraries which can be difficult to find - let alone diagnose and correct. To address this issue, direct comparisons between systematics and the evaluated files are indispensable tools for verification. A UKAEA report [14], which draws upon the systematics generated during the EAF development [15], has probed the larger landscape of TENDL reaction channels. These largely found agreement with the legacy systematics, except where the simplified nature of those systematics failed to represent the basic physics. This can be easily seen in Fig. 6, where lack of shell or even-odd effects are clearly evident. The lack of alternative channel competition, notably the (n,2p) channel which is opened up in the proton-rich nuclides far from stability, also
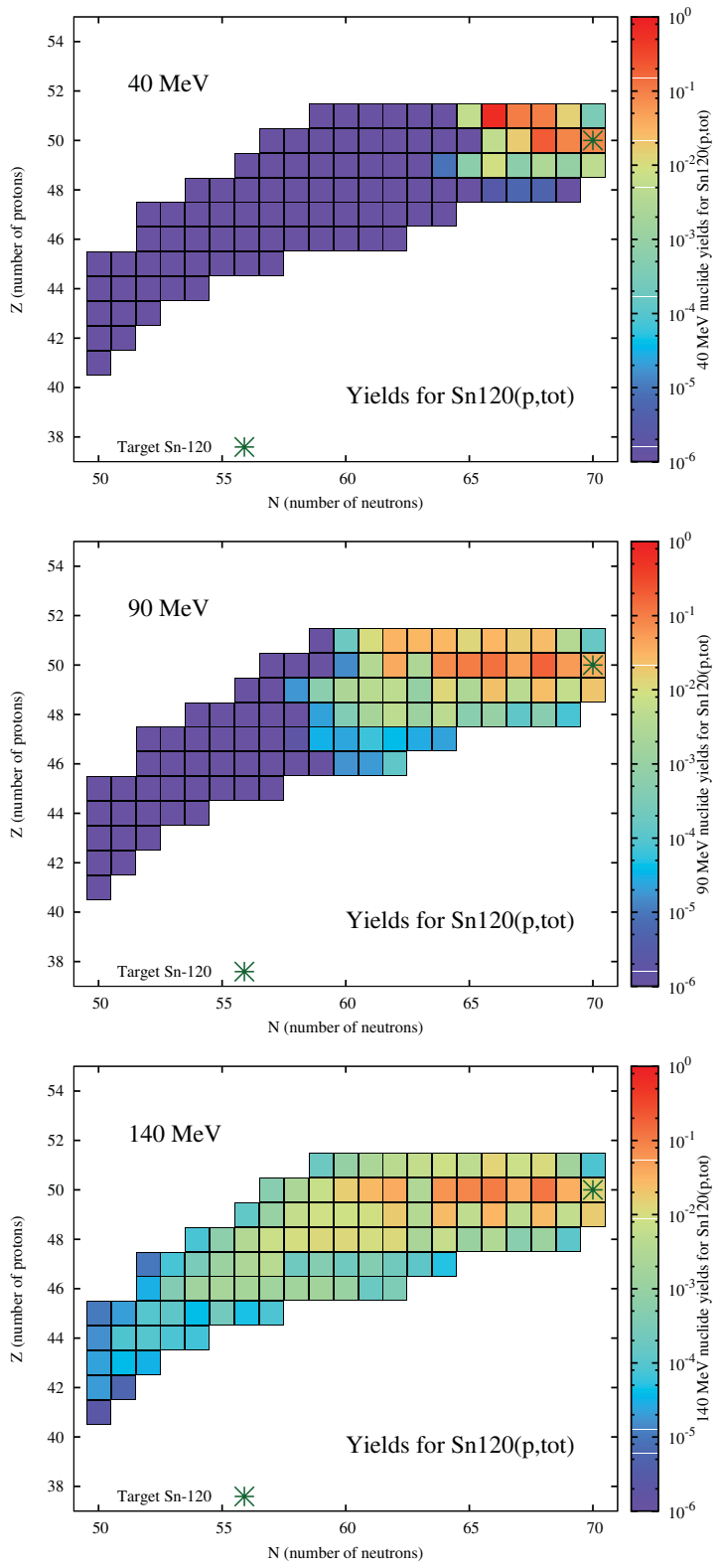

Figure 4. TENDL-2015 total residual nuclide production cross sections from proton-induced reactions on ${ }^{120} \mathrm{Sn}$. Incident energies for 40,90 , and $140 \mathrm{MeV}$ are shown to demonstrate the range of yields over $30-200 \mathrm{MeV}$.

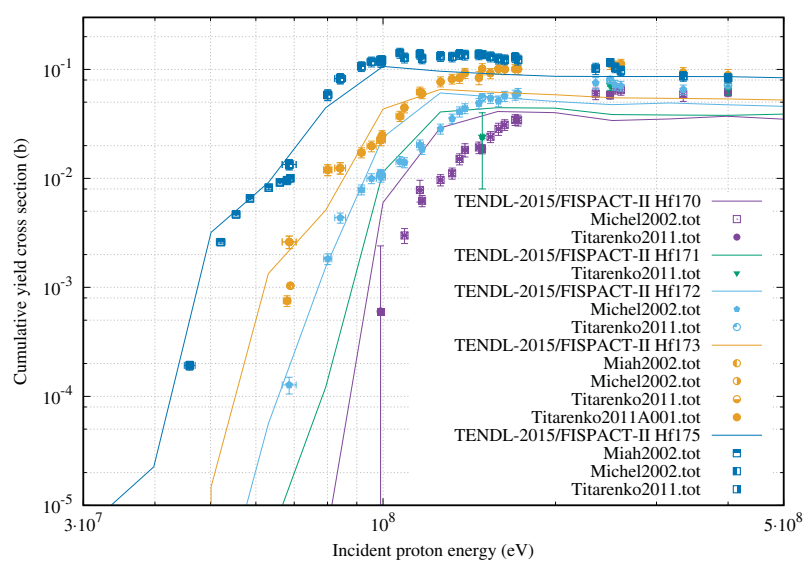

Figure 5. Cumulative TENDL-2015 proton-induced yields for ${ }^{n a t} \mathrm{~W}$ as calculated using JEFF-3.1.1 decay data with FISPACT-II over energies up to $500 \mathrm{MeV}$. 

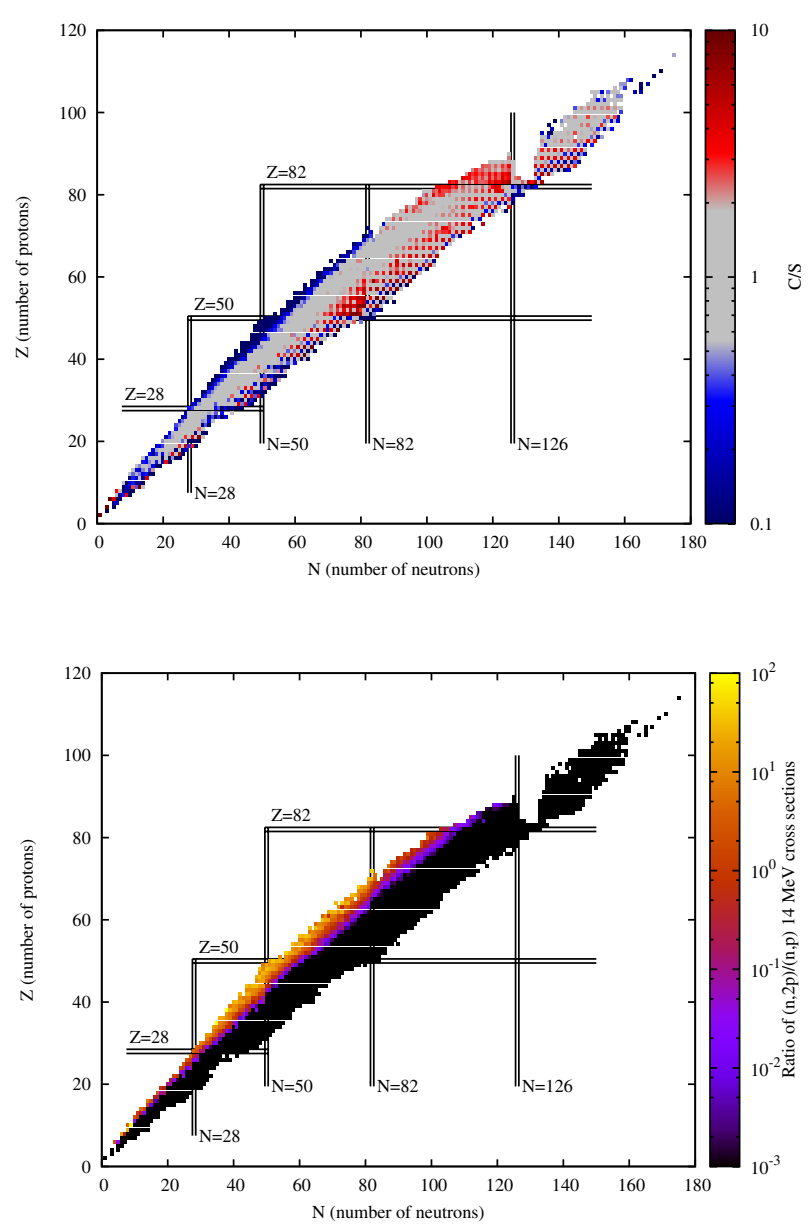

Figure 6. Nuclide chart showing TENDL-2014 (n,p) calculation to systematic (C/S) ratio (top) and ratio of $14 \mathrm{MeV}(\mathrm{n}, 2 \mathrm{p})$ to $(\mathrm{n}, \mathrm{p})$ cross sections (bottom).

prevents the calculation to systematic ratios $(\mathrm{C} / \mathrm{S})$ from providing a meaningful verification in those regions. However the systematics, where relevant, show generally good agreement. Along with the systematic checks over the whole of the TENDL target sets, a series of verification checks were performed against a UKAEA compilation of reference thermal and resonance integral measurements taken from the well-known sources $[16,17]$. These found very good agreement with some minor errors within the TENDL-2014 input parameter libraries that have been corrected for future distributions.
This work was funded by the RCUK Energy Programme [grant number EP/I501045]. To obtain further information on the data and models underlying this paper please contact PublicationsManager@ccfe.ac.uk.

\section{References}

[1] J.C. Sublet, J. Eastwood, J. Morgan, M. Fleming, M. Gilbert, Tech. Rep. UKAEA-R(11)11 Issue 8, UKAEA (2016)

[2] A. Koning, D. Rochman, Nuclear Data Sheets 113, 2841 (2012)

[3] F. Maekawa, Y. Ikeda, Fusion Engineering and Design 47, 377 (2000)

[4] R. Forrest, M. Pillon, J. Kopecky, A. Klix, S.P. Simakov, J.C. Sublet, P. Bém, M. Honusek, E. Šimecková, Tech. Rep. UKAEA-FUS-547, United Kingdom (2008)

[5] J.C. Sublet, M. Gilbert, Tech. Rep. CCFE-R(15)25, UKAEA (2015)

[6] M. Fleming, J.Ch. Sublet, J. Kopecky, Tech. Rep. CCFE-R(15)27, CCFE (2015)

[7] J. Kopecky, Tech. Rep. INCD(NED)-011, JUKO (2012)

[8] J. Kopecky, Tech. Rep. JEF/DOC-1575, JUKO (2014)

[9] J.-Ch. Sublet, M. Fleming, Tech. Rep. CCFER(15)29, UKAEA (2015)

[10] I. Dillmann, M. Heil, F. Käppeler, T. Rauscher, KADoNiS v0.3, in Proceeding of the workshop EFNUDAT Fast Neutrons (Geel, Belgium, 2009)

[11] Z. Bao, H. Beer, F. Käppeler, F. Voss, K. Wisshak, T. Rauscher, Atomic Data and Nuclear Data Tables 76, 70 (2000)

[12] T. Nakagawa, S. Chiba, T. Hayakawa, T. Kajino, Atomic Data and Nuclear Data Tables 91, 77 (2005)

[13] D. Rochman, A.J. Koning, J. Kopecky, J.-Ch. Sublet, P. Ribon, M. Moxon, Annals of Nuclear Energy 51, 60 (2013)

[14] M. Fleming, J.Ch. Sublet, J. Kopecky, D. Rochman, A. Koning, Tech. Rep. UKAEA-R(15)30, UKAEA (2015)

[15] R. Forrest, J. Kopecky, Fusion Engineering and Design 82, 73 (2007)

[16] S.F. Mughabghab, Atlas of Neutron Resonances: Resonance Parameters and Thermal Cross Sections. $\mathrm{Z}=1-100,5$ th edn. (Elsevier, 2006)

[17] W.M. Haynes, ed., Handbook of Chemistry and Physics, 96th edn. (CRC Press, 2015) 